\title{
An Angular Position-Based Two-Stage Friction Modeling and Compensation Method for RV Transmission System
}

\author{
Chao QI, Xianliang JIANG, Hongbo LIAO, Dapeng FAN, Sheng ZHANG
}

\begin{abstract}
In RV transmission system (RVTS), friction is closely related to rotational speed and angular position. However, classical friction models do not consider the influence of angular position on friction, resulting in limited accuracy in describing the RVTS frictional behavior. For this reason, this paper proposes an angular positionbased two-stage friction model for RVTS, and achieves a more accurate representation of friction of RVTS. The proposed model consists of two parts, namely pre-sliding model and sliding model, which are divided by the maximum elastic deformation recovery angle of RVTS obtained from loading-unloading tests. The pre-sliding friction behavior is regarded as a spring model, whose stiffness is determined by the angular position and the acceleration when the velocity crosses zero, while the sliding friction model is established by the angular-segmented Stribeck function, and the friction parameters of the adjacent segment are linearly smoothed. A feedforward compensation based on the proposed model was performed on the RVTS, and its control performance was compared with that using the classical Stribeck model. The comparison results show that when using the proposed friction model, the low-speed-motion smoothness of the RVTS can be improved by $14.2 \%$, and the maximum zero-crossing speed error can be reduced by $37.5 \%$, which verifies the validity of the proposed friction model, as well as the compensation method.
\end{abstract}

Keywords: angular position; friction modeling; low-speed-motion smoothness; maximum elastic deformation recovery angle; RV transmission system (RVTS); zero-crossing speed error

\section{INTRODUCTION}

$\mathrm{RV}$ reducer has many outstanding features such as small size, lightweight, and large reduction ratio, so it has been widely used in the joints of industrial robots. Improving the servo performance of RV transmission system (RVTS) is of great importance for the accurate motion of robot joint. However, friction, as a nonnegligible nonlinear factor in RVTS, causes the system to stick and slip at low speed, and results in a large zerocrossing speed error. Therefore, accurate observation and compensation of friction is necessary for improving the RVTS servo performance.

The exploration of frictional behavior has been taken for hundreds of years. However, due to its complex dynamics, current models cannot completely describe the frictional behaviors yet. The friction models include static models and dynamic models [1]. The commonly used static friction models, such as Coulomb model, Dahl model and Stribeck model, have relatively simple structures and few parameters, hence the parameter identification is convenient. However, static models cannot accurately represent the pre-sliding frictional behavior. Therefore, the speed tracking error cannot be eliminated when the speed approaches zero. Compared with static models, dynamic models, e.g. LuGre model [2] and GMS model [3], have a better presentation in the pre-sliding frictional behavior. However, since the dynamic models are complex in structure, parameter identification is difficult. As a result, they are rarely used in practice.

The models mentioned above are established for frictional behavior description under general conditions. Meanwhile, many studies have also been conducted on the friction dynamics of RVTS. Lv [4] constructed a precision $\mathrm{RV}$ transmission test bench and used the classical Stribeck model to fit the friction data obtained under no-loading conditions. So it is a traditional friction modeling method only considering rotating speed. Wu [5] studied the influence of load on the frictional behavior of RVTS and found that both static friction and Coulomb friction components are linear to the load torque. Hence an improved Stribeck friction model with load torque parameters was proposed. However, due to the limited number and type of sensors, load torque cannot be observed accurately in real time, so it is hard to estimate RVTS friction using the load torque value. Yu et al. [6-9] made detailed mechanical analysis of the main friction sources in RV reducer. They found that assembly accuracy and unbalanced mass have a large impact on RVTS friction. However, since these methods involve structural and material parameters, their conclusions cannot be used directly to design a friction compensation method. From the above studies, it can be seen that the RVTS friction torque is affected by multiple factors that cannot be directly measured, such as assembly accuracy, unbalanced mass, load torque and materials. Actually, these factors can be regarded as the effect of angular position on friction [10]. So if the relationship between the angular position and the RVTS friction can be established, the accuracy of the friction model will be improved to achieve higher motion performance.

Since mechanical friction behaves significantly different under low speed and high speed conditions, the friction model is usually divided into two parts, namely pre-sliding model and sliding model.[11] The research on the relationship between angular position and friction mainly concentrates on the devices that are equipped with a harmonic reducer or a ball screw. In sliding regime, Taghirad et al. [12] found that there is a special trigonometric relationship between friction and angular position in harmonic reducer, so he built an improved Stribeck friction model containing several position-related sinusoidal components. Cong et al. [13] established a model considering friction, rotation speed, angular position and direction of an ISP direct-drive system. Li et al. [14] segmented the working stroke of a $\mathrm{CNC}$ guide and identified the Stribeck friction model of each segment. However, due to the low signal-to-noise ratio of the current signal, the number of angle segments is limited. Moreover, the parameters of the friction model between adjacent 
segments are not continuous, which is one of the main reasons that limit the accuracy of this friction model. Although the above researches come from different mechanisms, they are valuable for constructing the sliding friction model of RVTS. In pre-sliding regime, the motor speed is always in the neighborhood of zero. To obtain the actual speed accurately, it is necessary to assemble an encoder with high resolution. However, for the assembly equipped with an encoder owning a large measurement range, the speed obtained by differentiating encoder data is of low resolution, so it is not possible to use such an encoder for observing the pre-sliding speed. As a result, it seems necessary to establish the pre-sliding friction model in combination with the mechanical properties of the assembly. Liao et al. [15] and Aung et al. [17] used ramp signals to perform torque loading and unloading tests on harmonic drive assemblies. He found that the maximum elastic deformation recovery angle of a harmonic reducer does not change with the loading rate and angular position. Accordingly, a friction compensation scheme based on angular relationship is proposed. Yang et al. [16] observed that the break-away displacement of the $\mathrm{CNC}$ spindle guide is constant, so he combined the constant break-away displacement with the acceleration at zero-crossing speed to obtain the break-away speed for distinguishing the sliding regime from the pre-sliding regime. Combined with the Stribeck model in the sliding regime, a two-stage friction model of the $\mathrm{CNC}$ guide with only the velocity signal as the input is established. Presently, there are few researches on the relationship between pre-sliding friction and angular position of RVTS, but similar to harmonic drive assemblies and CNC spindle guides, RVTS also requires a large-scale turning, thus the speed obtained by the encoder cannot be directly used for pre-sliding friction modeling. So it is also necessary to study the loadingunloading characteristics of the RVTS in the pre-sliding regime.

The rest parts of the paper are organized as follows: Section 2 presents a two-stage friction modeling method that can accurately describe the friction behavior of RVTS. Section 3 introduces the identification process of the proposed model, and verifies the effectiveness of the proposed friction model by several experiments. In addition, the performance of the controller combined with friction compensation and DOB is also discussed. In Section 4, the last part of the paper, some remarks and conclusions are given.

\section{FRICTION MODELING OF RVTS}

A typical RV transmission system is composed of an incremental encoder, a RV reducer, an optical encoder, an $\mathrm{AC}$ servo motor and a driver, as shown in Fig. 1. The AC driver operates in torque mode or speed mode, and outputs a voltage that is proportional to the actual torque. An incremental encoder with a 10,000-line angular resolution is mounted on the motor shaft to obtain the angle of $\mathrm{AC}$ servo motor. The reduction ratio of the RV reducer ZKRV$20 \mathrm{E}$ is 161 , and the optical encoder with 17-bit angular resolution is fixed on the load shaft to obtain the angular position of RVTS. In addition, dSPACE1104 is used as the hardware-in-loop simulation platform for designing and verifying the control algorithms for RVTS. The input of
dSPACE1104 comes from the incremental encoder, the optical encoder and the driver, and the output is a control voltage of the driver with an operation period of $0.001 \mathrm{~s}$.

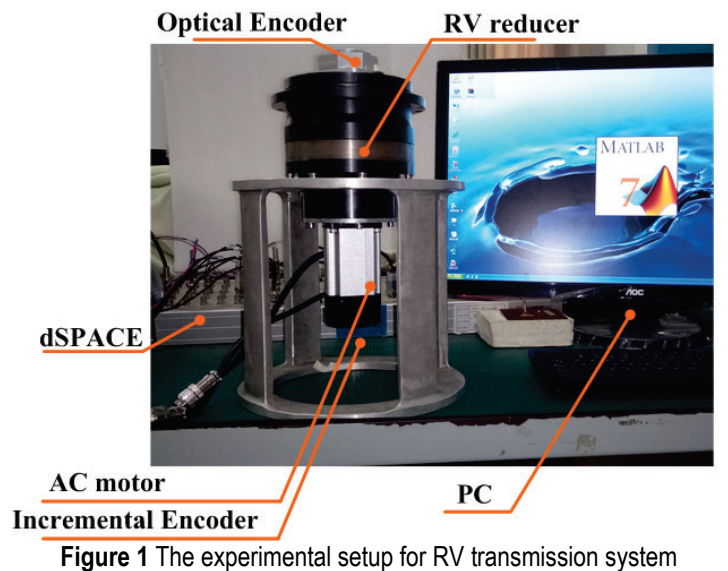

In order to describe the frictional behavior in entire operating speed, the friction model is usually divided into a pre-sliding regime and a sliding regime, respectively. The pre-sliding regime represents the state where the assembly rotates in the vicinity of zero speed and no significant rotation occurs, while the sliding regime represents the friction behavior when it rotates obviously. The two regimes are differentiated by the break-away speed $\omega_{\text {break }}$ as

$T_{\mathrm{f}}= \begin{cases}T_{\text {preslide }} & |\omega|<\omega_{\text {break }} \\ T_{\text {slide }} & |\omega|>\omega_{\text {break }}\end{cases}$

As can be seen from Eq. (1), in order to establish the friction model $T_{\mathrm{f}}$, it is necessary to determine the breakaway speed $\omega_{\text {break }}$, and model the pre-sliding friction $T_{\text {preslide }}$ as well as the sliding friction $T_{\text {slide. }}$ So the following will introduce the friction modeling method proposed in this paper.

\subsection{Classical Stribeck Model}

As a widely used friction model, the Stribeck model can well represent the effect that the friction decreases with increasing speed at low speed rotation, as shown in Fig. 2(a).The expression of the curve in Fig. 2(a) is

$$
T_{\text {slide }}(\omega)=T_{c}^{ \pm}+\left(T_{s}^{ \pm}-T_{c}^{ \pm}\right) \cdot e^{-\left(\frac{\omega}{\Omega_{1}^{ \pm}} \delta_{ \pm}\right.}+B^{ \pm} \cdot \omega, \omega \neq 0
$$

where $T_{c}^{ \pm}, T_{s}^{ \pm}, \Omega_{1}^{ \pm}, \delta^{ \pm}, B^{ \pm}$represent Coulomb friction, maximum static friction, Stribeck velocity, deformation coefficient and viscous friction coefficient. In addition, the superscript + and - respectively represent anticlockwise and clockwise rotation.

In model (2), the viscous friction coefficient $B^{ \pm}$is a constant. However, in many cases, such as RVTS, viscous friction coefficient $B^{ \pm}$gradually decreases as the rotational speed $\omega$ increases, as shown in Fig. 2(b). In order to deal with this effect, an improved Stribeck model whose viscous friction coefficient changes with the speed has been proposed in [18]. The expression is shown as 


$$
\begin{aligned}
& T_{\text {slide }}(\omega)= \\
& =T_{c}^{ \pm}+\left(T_{s}^{ \pm}-T_{c}^{ \pm}\right) \cdot e^{-\left(\frac{\omega}{\Omega_{1}^{ \pm}}\right)^{\delta_{ \pm}}}+B^{ \pm} \cdot e^{-\left(\frac{\omega}{\Omega_{2}^{ \pm}}\right)} \cdot \omega, \omega \neq 0
\end{aligned}
$$

where $\Omega_{2}^{ \pm}$is added to indicate the rapidity of variation of viscous friction coefficient $B^{ \pm}$with speed $\omega$.

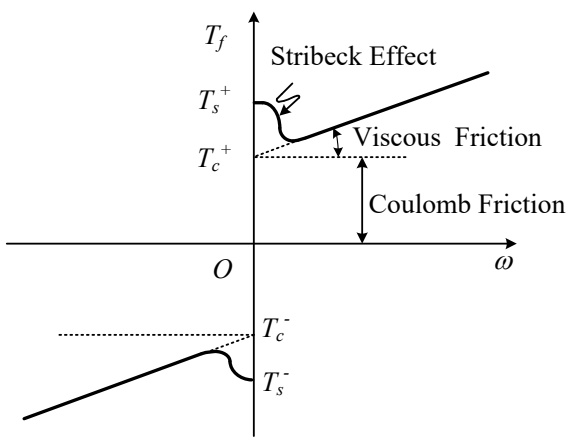

(a)
With the help of model (3), one can estimate the average friction torque when RVTS rotates in a constant speed $\omega$. However, the disadvantage of model (3) is that it fails to represent the frictional behavior in the pre-sliding regime. Moreover, since angular position is not considered in this model, the representation accuracy of the real-time friction behavior in sliding regime is also limited.

Figure 2 Stribeck curve with (a) a constant viscous friction coefficient; (b) a speed-dependent viscous friction coefficient.

\subsection{Proposed Friction Model 2.2.1 Pre-sliding Friction Modeling}

Make the servo driver operate in torque mode, and then use the ramp command $T_{\text {ref }}$ shown in (4) to load the RVTS. Set the loading rate $r=0.06 \mathrm{Nm} / \mathrm{s}$, and sample the incremental encoder angle by dSPACE simultaneously. By adjusting the maximum torque value $T_{\max }$ in (4), one can have the experimental curve shown in Fig. 3.

$T_{\text {ref }}=\left\{\begin{array}{ll}r t & t<\frac{T_{\max }}{r} \\ 0 & \text { otherwise }\end{array}\right.$.

It can be seen from Fig. 3 that when the loading angle does not exceed six pulses (i.e., six angular resolutions), the recovery angle equals the loading angle, which means that the RVTS can always return to the initial loading position. However, once the loading angle exceeds six pulses, RVTS cannot return to its original position any longer due to sliding that happens.

Furthermore, by performing multiple loadingunloading tests with different angular positions and different loading rates, it can be seen that the maximum elastic deformation recovery angle of RVTS keeps

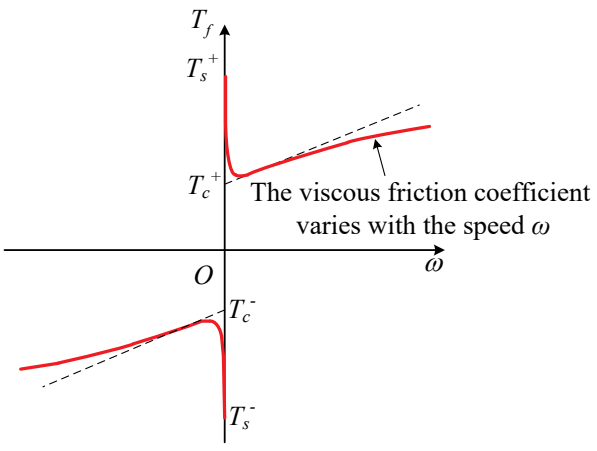

(b) constant. Therefore, it is reasonable to use 6 angular resolutions as the break-away displacement to distinguish the pre-sliding and sliding regime. So the break-away displacement $\theta_{\text {break }}$ can be obtained as

$\theta_{\text {break }}=6 \theta_{\text {res }}$,

where $\theta_{\text {res }}$ denotes one angular resolution of the incremental encoder.

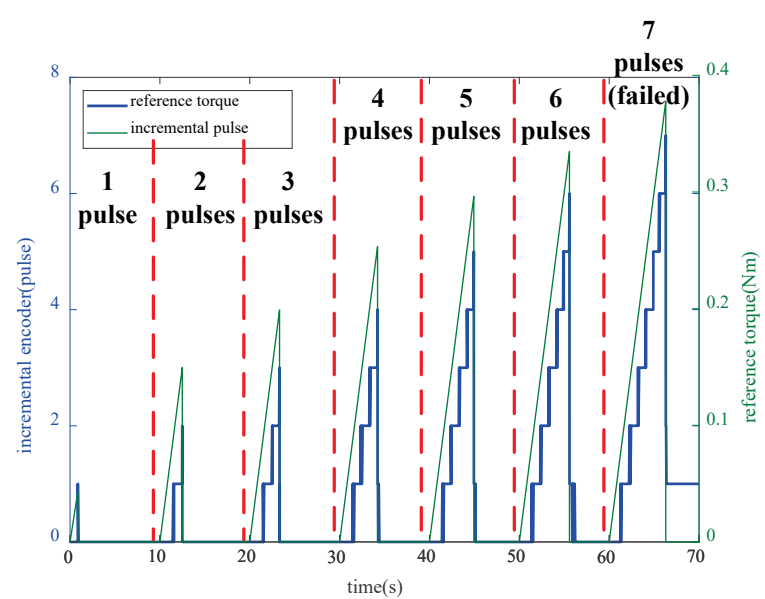

Figure 3 The experimental curve of loading-unloading test for RVTS.

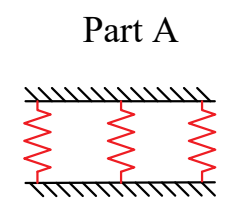

Part B

$$
\Delta \theta=0
$$

Presliding Regime

(a)

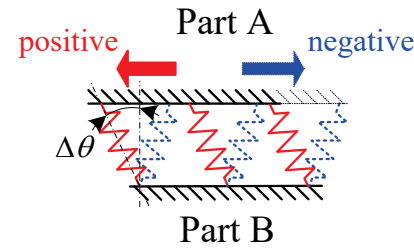

$$
|\Delta \theta| \leq \theta_{\text {break }}
$$

Presliding Regime

$$
\text { (b) }
$$

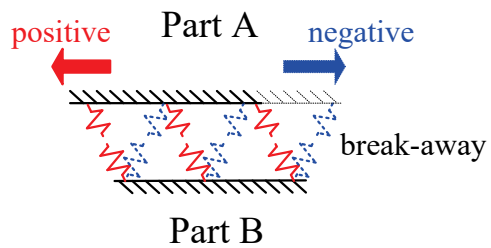

$|\Delta \theta|>\theta_{\text {break }}$

Sliding Regime

(c)

Figure 4 The spring model for pre-sliding friction behavior of RVTS while the relative displacement $\Delta \theta$ is (a) equivalent to zero; (b) less than break-away displacement $\theta_{\text {break }}$ and (c) larger than $\theta_{\text {break. }}$ 
The relationship between friction and angular displacement in the pre-sliding regime can be regarded as a spring model, as shown in Fig. 4. When the relative displacement of the two contact surfaces, Part A and Part $\mathrm{B}$, is less than $\theta_{\text {break, }}$, the contact surfaces are elastically connected, and the elastic force between the two contact surfaces is pre-sliding friction. However, when the relative displacement becomes larger than $\theta_{\text {break, }}$ the spring breaks and sliding happens, then the friction between the two contact surfaces no longer matches with the spring model.

Suppose the stiffness of the spring model is $K$, and the relative displacement is $\Delta \theta$, then the pre-sliding friction $T_{\text {preslide }}$ can be expressed as

$T_{\text {preslide }}=K \cdot \Delta \theta,|\Delta \theta| \leq \theta_{\text {break }}$.

Since the pre-sliding regime is usually of a narrow scale, it can be considered that the acceleration remains constant in the pre-sliding regime [16]. Assuming that the angular acceleration is $a_{0}$ when speed crosses zero, the break-away velocity $\omega_{\text {break }}$ can be obtained as

$\omega_{\text {break }}=\sqrt{2 a_{0} \theta_{\text {break }}}$.

If the sliding friction model $T_{\text {slide }}$ is known, the breakaway torque $T_{\text {break }}$ could be derived as

$$
T_{\text {break }}=T_{\text {slide }}\left(\theta, \omega_{\text {break }}\right)=\left.T_{\text {preslide }}\right|_{\Delta \theta=\theta_{\text {break }}} .
$$

Substitute Eqs. (7), (8) into (6), and let $\Delta \theta=\theta_{\text {break, then }}$ $K$ can be derived as

$$
K=T_{\text {slide }}\left(\theta, \sqrt{2 a_{0} \theta_{\text {break }}}\right) / \theta_{\text {break }}=K\left(\theta, a_{0}\right) .
$$

In Eq. (9), the angular position $\theta$ can be obtained directly by the optical encoder, and the acceleration $a_{0}$ can be estimated by differentiating the reference speed. If only the sliding friction model $T_{\text {slide }}$ is known, then the value of $K$ could be obtained. So next section will present a detailed modeling process for the sliding friction $T_{\text {slide }}(\theta, \omega)$.

\subsubsection{Sliding Friction Modeling}

Due to the combined effects of material and structural factors, the sliding friction of RVTS varies with the angular position. Instead of the model (2) and (3), this section provides an angular-segmented Stribeck friction modeling method to represent the angular distribution of friction in RVTS. The modeling procedures are as follows:

\section{Step 1. Determine the zero position of the load side and divide a circle into $N$ segments}

Determine the zero position of the load according to the angle obtained from the optical encoder, and divide a circle (i.e., $2 \pi$ radians) into $N$ segments. Then mark each segment from 1 to $N$ in the positive direction (i.e., anticlockwise), as shown in Fig. 5. Thus the angle range represented by the $k^{\text {th }}$ segment $\left(k \in[1, N] \mid k \in \mathbb{Z}_{+}\right)$is $\left[\theta_{k}\right.$, $\left.\theta_{k+1}\right)$, where $\theta_{k}$ equals $(k-1) \cdot 2 \pi / N$ radians and $\theta_{k+1}$ equals $2 \pi k / N$ radians.

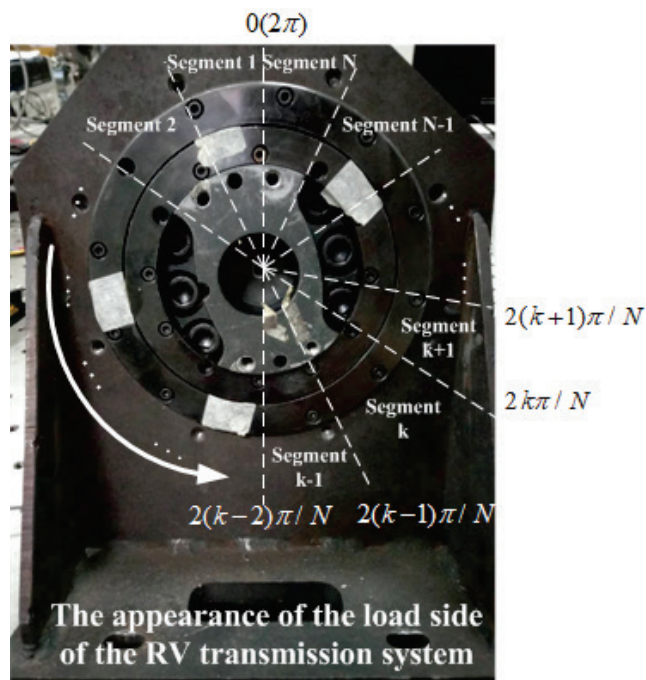

Figure 5 Determine the zero position of RVTS, and divide a full circle of the load side into $\mathrm{N}$ segments.

Step 2. Obtain the correspondence among segment, speed and friction torque

First, construct a speed close loop with the speed value measured by the incremental encoder. Subsequently, make RVTS rotate a full circle at a set of constant speeds $\omega \in$ $\left\{\omega_{1}, \ldots, \omega_{i}, \omega_{i+1}, \ldots, \omega_{M-1}, \omega_{M} \mid\right.$ if $\left.1 \leq i \leq M-1,\left|\omega_{i}\right|<\left|\omega_{i+1}\right|\right\}$, collecting the torque starting from the zero position determined in Step 1 each time. Then, average the torque at each rotational speed segment by segment to obtain the correspondence among segment, rotational speed and average friction torque. Therefore, we can mark the calculated average friction torque of the $k^{\text {th }}$ segment as $T_{k, i}$ while the $i^{\text {th }}$ rotational speed of RVTS is $\omega_{i}$, as is shown in Fig. 6.

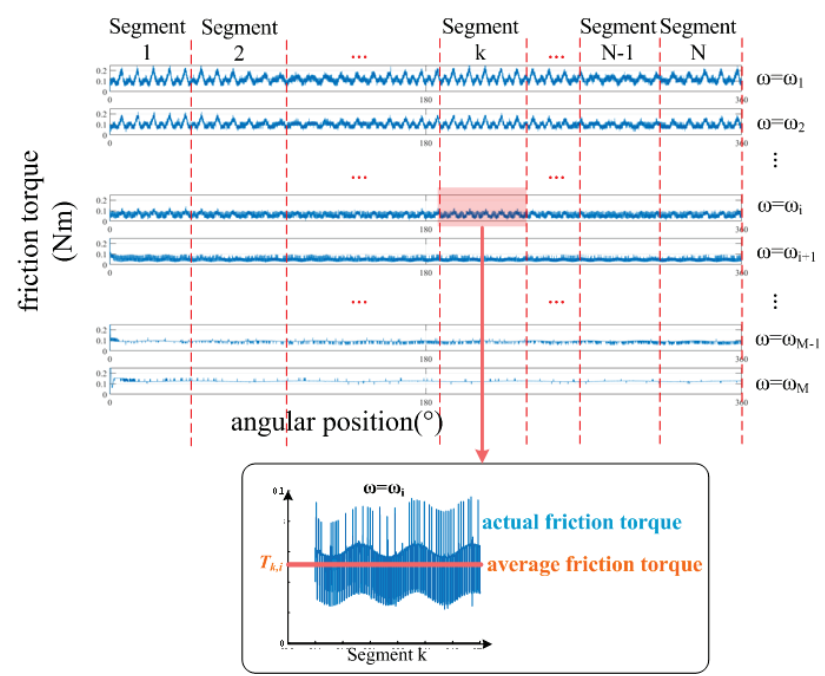

Figure 6 According to the actual friction data collected in the speed mode of RVTS, the average friction torque of all $N$ segments at all $M$ given speeds can be calculated. Denote $T_{k, \mathrm{i}}$ as the average friction in the $k^{\text {th }}$ segment at the $i^{\text {th }}$ rotational speed $\omega_{i}$.

\section{Step 3. Identify friction parameters for all segments}

In the $k^{\text {th }}$ segment, identify the parameters of Stribeck model (3) with the speed $\omega_{i}$ and average torque $T_{k, i}$ acquired in Step 2 by nonlinear least-squares method. The speed and torque values for the remaining segments can also be processed accordingly. Thus, the Stribeck model parameters can be totally obtained for all angular segments. 
The fitted Stribeck curves for Segment 1 to $N$ are respectively shown in Fig. 7.

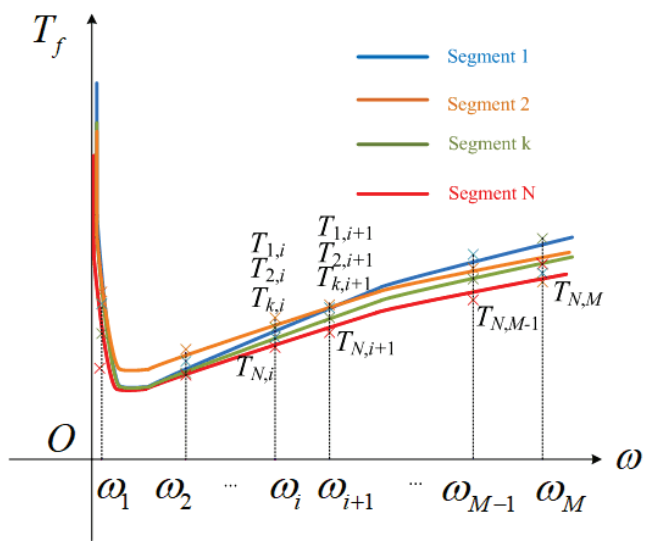

Figure 7 The fitted Stribeck curve by nonlinear least-square method for the average frictions of all segments.

Step 4. Linearize the friction model parameters with the angular position

In order to ensure that the sliding friction model is continuous with angular position, the identified parameters of adjacent segments need to be linearized. Suppose the parameters identified in the $\mathrm{k}$-th and $\mathrm{k}+1$-th segments are respectively $\left(T_{c, k}^{ \pm}, T_{s, k}^{ \pm}, \Omega_{1, k}^{ \pm}, \delta_{k}^{ \pm}, B_{k}^{ \pm}, \Omega_{2, k}^{ \pm}\right) \quad$ and $\left(T_{c, k+1}^{ \pm}, T_{s, k+1}^{ \pm}, \Omega_{1, k+1}^{ \pm}, \delta_{k+1}^{ \pm}, B_{k+1}^{ \pm}, \Omega_{2, k+1}^{ \pm}\right)$, then for any angular position $\theta \in\left[\theta_{k}, \theta_{k+1}\right)$, the corresponding model parameters $\left(T_{c, \theta}^{ \pm}, T_{s, \theta}^{ \pm}, \Omega_{1, \theta}^{ \pm}, \delta_{\theta}^{ \pm}, B_{\theta}^{ \pm}, \Omega_{2, \theta}^{ \pm}\right) \quad$ can be obtained by

$$
\left[\begin{array}{l}
T_{c, \theta}^{ \pm} \\
T_{s, \theta}^{ \pm} \\
\Omega_{1, \theta}^{ \pm} \\
\delta_{\theta}^{ \pm} \\
B_{\theta}^{ \pm} \\
\Omega_{2, \theta}^{ \pm}
\end{array}\right]=\frac{N}{2 \pi} \cdot\left[\begin{array}{cc}
T_{c, k}^{ \pm} & T_{c, k+1}^{ \pm} \\
T_{s, k}^{ \pm} & T_{s, k+1}^{ \pm} \\
\Omega_{1, k}^{ \pm} & \Omega_{1, k+1}^{ \pm} \\
\delta_{k}^{ \pm} & \delta_{k+1}^{ \pm} \\
B_{k}^{ \pm} & B_{k+1}^{ \pm} \\
\Omega_{2, k}^{ \pm} & \Omega_{2, k+1}^{ \pm}
\end{array}\right] \cdot\left[\begin{array}{l}
\theta_{k+1}-\theta \\
\theta-\theta_{k}
\end{array}\right],
$$

thus the sliding friction $T_{\text {slide }}(\theta, \omega)$ is

$$
\begin{aligned}
& T_{\text {slide }}(\theta, \omega)= \\
& =T_{c, \theta}^{ \pm}+\left(T_{s, \theta}^{ \pm}-T_{c, \theta}^{ \pm}\right) \cdot e^{-\left(\frac{\omega}{\Omega_{1, \theta}^{ \pm}}\right)^{\delta_{\theta}^{ \pm}}}+B_{\theta}^{ \pm} \cdot e^{-\left(\frac{\omega}{\Omega_{2, \theta}^{ \pm}}\right)} \cdot \omega
\end{aligned}
$$

Substituting (11) into (9), the stiffness coefficient of pre-sliding friction $K\left(\theta, a_{0}\right)$ can be solved.

\section{FRICTION COMPENSATION AND VERIFICATION}

Establish the friction model using the method introduced in Section 2.2, and make the model as the feedforward block to observe and compensate for friction, as is shown in Fig. 8. When the block detects that the actual speed is 0 , switch 1 is turned on, making the variable $\theta_{\text {memory }}$ equal to the actual angular position $\theta$; otherwise, switch 1 is turned off, and $\theta_{\text {memory }}$ remains the value at the moment that switch1 is triggered and will never change with the actual angular position $\theta$ until switch 1 turns on again. Subtract the actual angular position $\theta$ and the angle $\theta_{\text {memory, }}$ and the difference $\Delta \theta$ is used to enable switch2 to select the output of pre-sliding or sliding friction model. When $|\Delta \theta| \leq 6 Q_{\text {res, }}$, the block outputs the results of the pre-sliding friction model; otherwise, it outputs the results of the sliding friction model. Because of the obvious quantization noise in the actual velocity obtained by the incremental encoder, the reference speed is used as the input of the friction observer instead in order to ensure the smoothness of the motion.

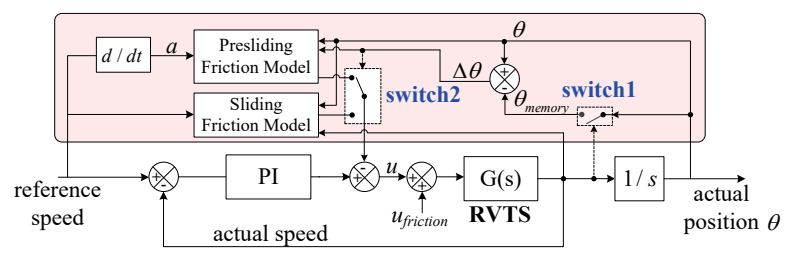

Figure 8 The feedforward control diagram based on the proposed friction model.

\subsection{Identification}

Since the pre-sliding friction parameters of the proposed friction model need to be determined by the sliding friction model, the identification process of sliding friction parameters will be mainly introduced in this section. Here, we divide a circle of the RVTS shown in Fig. 1 into 90 segments, and specify the zero position as well as moving direction. Then follow the step provided in section 2.2.2 to obtain the Stribeck friction parameters of the RVTS of each segment. The correspondence measured between the friction torque and the rotational speed of all 90 segments is shown in Fig. 9. As can be seen from this figure, the relationship between speed and friction matches the curve shown in Fig. 2(b). The identification values of parameters of the Stribeck model (3) for each angular segment are shown in Fig. 10, where the red and blue dotted lines represent the identification result of positive and negative direction, respectively.

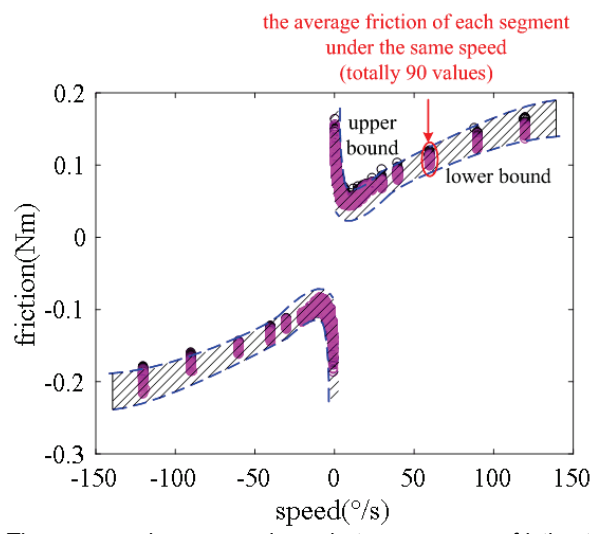

Figure 9 The measured correspondence between average friction torque and rotational speed of all 90 segments.

The fitting accuracy of each segment based on the Stribeck friction model (3) is represented by the index $R^{2}$, which is defined as [19] 


$$
R^{2}=\frac{\left[\sum_{i=1}^{n}\left(y_{i}-\bar{y}\right)^{2}-\sum_{i=1}^{n}\left(y_{i}-\hat{y}_{i}\right)^{2}\right]}{\sum_{i=1}^{n}\left(y_{i}-\bar{y}\right)^{2}}
$$

where $y_{i}$ is the actual friction torque, $\bar{y}$ is the average friction torque, and $\hat{y}_{i}$ is the fitting value of the model. $R^{2}$ values between 0 and 1 , and if the fitting accuracy of the function is higher, the $R^{2}$ will be closer to 1 .
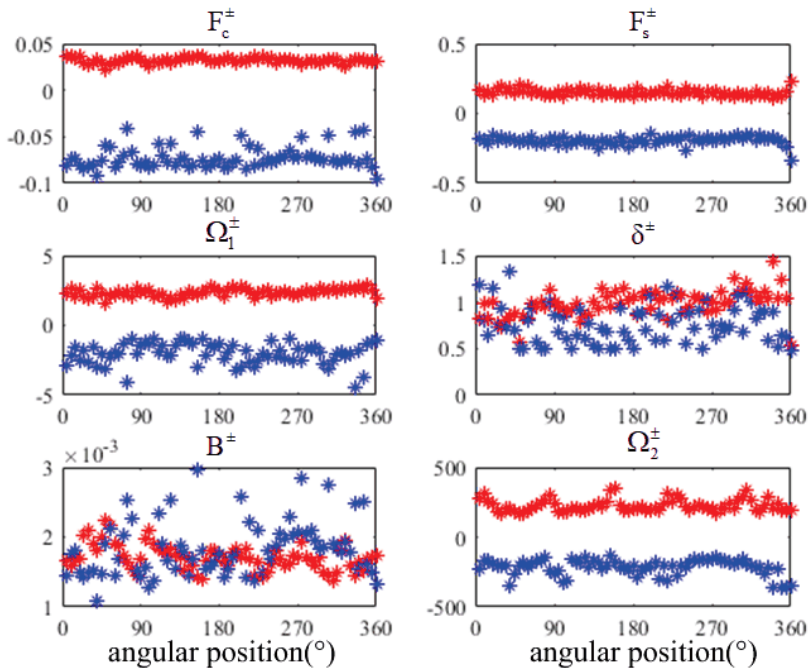

Figure 10 Identified parameter results of the Stribeck model (3) for all 90 segments.

The fitting accuracy of each segment is shown in Fig. 11, from which can be seen that the forward fitting accuracy $R^{2}>98 \%$, and the negative fitting precision $R^{2}>$ $95.4 \%$, thus the overall fitting precision is satisfactory. As a result, the identification results above can be used in the observation and compensation of friction. In order to test the validity of the proposed friction model, it is necessary to carry out an experimental comparison of friction compensation effects, which will be described in Section 3.2 .

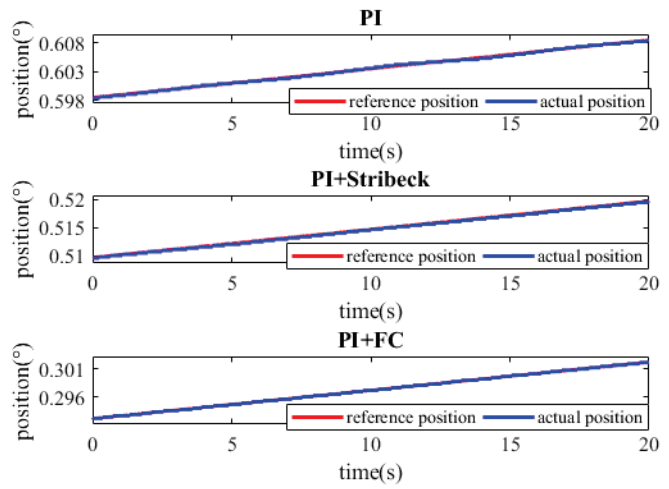

(a)

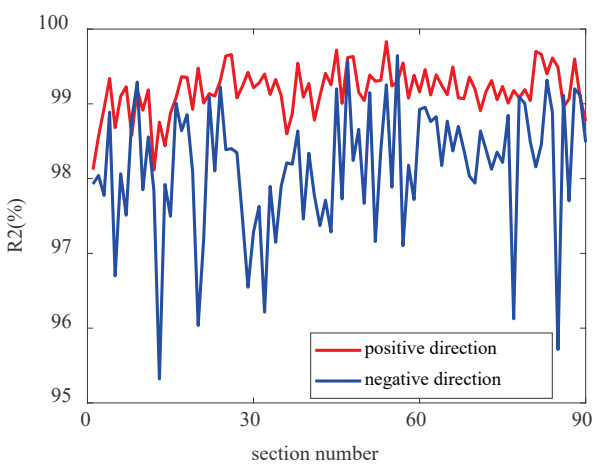

Figure 11 Fitting accuracy of the Stribeck model (3) for all 90 segments.

\subsection{Experimental Verification}

In this section, three control methods, i.e., PI with no feedforward compensation (PI), PI with the classical Stribeck model-based feedforward compensation (PI+Stribeck), and PI with the proposed friction modelbased feedforward compensation (PI+FC), are used to compare the low-speed-motion smoothness and the zerocrossing speed error of the RVTS in order to verify the effectiveness of the proposed friction model in this paper.

\subsubsection{Comparison of Low-Speed-Motion Smoothness}

Set the RVTS to be position closed loop with a ramp function as the reference position, and a proportional controller is adopted in the position loop. Meanwhile, PI, $\mathrm{PI}+$ Stribeck and $\mathrm{PI}+\mathrm{FC}$ are used as the speed loop controller respectively. The P and PI controller adopted in the speed loop and the position loop remain unchanged in the three control methods, and the controller parameters are listed in Tab. 1.

Table 1 Parameters of the P and PI controller used in the position and speed

\begin{tabular}{|c|c|c|c|}
\multicolumn{4}{|c|}{ loop } \\
\hline$r$ & $K_{\mathrm{pp}}{ }^{1}$ & $K_{\mathrm{sp}}{ }^{1}$ & $K_{\mathrm{si}}{ }^{1}$ \\
\hline $0.0005^{\circ} / \mathrm{s}$ & 5 & 0.3 & 20 \\
\hline
\end{tabular}

${ }^{1} K_{\mathrm{pp}}$ is the proportional gain of position loop, $K_{\mathrm{sp}}$ is the proportional gain of speed loop, and $K_{\mathrm{si}}$ is the integral gain of speed loop.

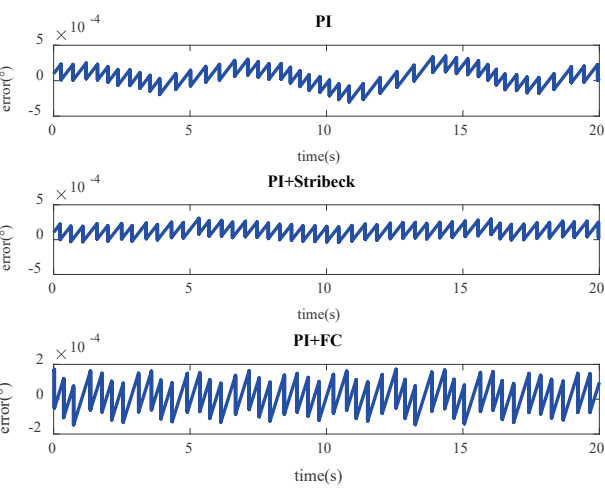

(b)

Figure 12 The actual position and angular tracking error with $\mathrm{PI}, \mathrm{PI}+\mathrm{Stribeck}$ and $\mathrm{PI}+\mathrm{FC}$ as the speed controller (the slope of the reference position $r=0.0005 \%$ s).

In order to effectively evaluate the smoothness of low speed motion with different control methods, the angular fluctuation rate $\varepsilon$ can be used to quantify the low-speedmotion smoothness as

$$
\varepsilon=\Delta \theta_{\mathrm{pp}} / \theta_{\text {ref }}
$$

where $\theta_{\text {ref }}$ represents the variation of the ramp position, and $\Delta \theta_{\mathrm{pp}}$ is the peak-to-peak value of the tracking error. Obviously, for the same displacement $\theta_{\text {ref, }}$ if the speed 
fluctuates more obviously, $\Delta \theta_{\mathrm{pp}}$ will be larger. Hence the value of $\varepsilon$ will increase. Ideally, when the assembly rotates in an uniform velocity, $\Delta \theta_{\mathrm{pp}}=0$, then $\varepsilon$ equals 0 exactly.

When the slope of the reference angle is $0.0005 \%$, the actual position and tracking errors of the three control methods are shown in Fig. 10.

It can be measured from Fig. 12 that $\Delta \theta_{\mathrm{pp}}$ is $6 \times 10^{-4 \circ}$, $3.5 \times 10^{-4 \circ}, 3.0 \times 10^{-4 \circ}$ when PI, PI+Stribeck and PI+FC are used as the speed controller respectively. In addition, the angular fluctuation rate $\varepsilon$ calculated according to (13) can be listed in Tab. 2 .

Table 2 the angular fluctuation rate using $\mathrm{PI}, \mathrm{PI}+\mathrm{Stribeck}$ and $\mathrm{PI}+\mathrm{FC}$ as the

\begin{tabular}{|c|c|c|c|}
\hline speed controller \\
\hline Control method & PI & PI+Stribeck & PI+FC \\
\hline Angular fluctuation rate $\varepsilon(\%)$ & 4.0 & 2.33 & 2.0 \\
\hline
\end{tabular}

PI

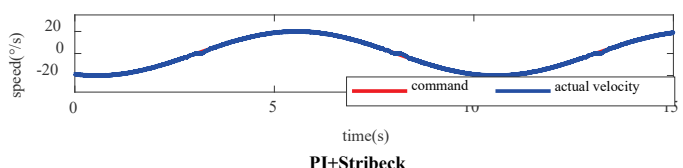

PI+Stribeck

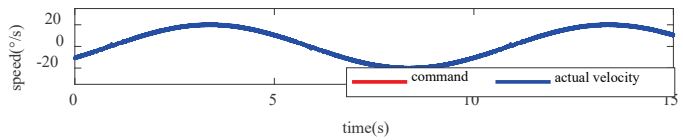

$\mathbf{P I}+\mathbf{F C}$

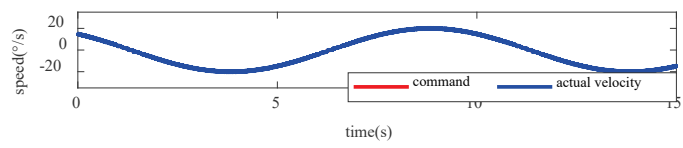

(a)
As can be seen from Tab. 2, comparing with the feedforward compensation method being based on the classical Stribeck model (3), the method being based on the proposed friction model can reduce the speed fluctuation by $14.16 \%$, which indicates that the proposed friction model has a higher accuracy in sliding regime.

\subsubsection{Comparison of Zero-Crossing Speed Error}

Set RVTS to speed mode (i.e., speed close loop) with $\mathrm{PI}$, PI+Stribeck and PI+FC as the speed controller respectively, and make the reference speed a sinusoidal signal with an amplitude of $20 \%$ s and a frequency of 0.1 $\mathrm{Hz}$. The parameters of PI controller remain the same as those listed in Tab. 1. Then the speed tracking error can be measured, as shown in Fig. 13. The zero-crossing speed errors by the three control methods are listed in Tab. 3 .

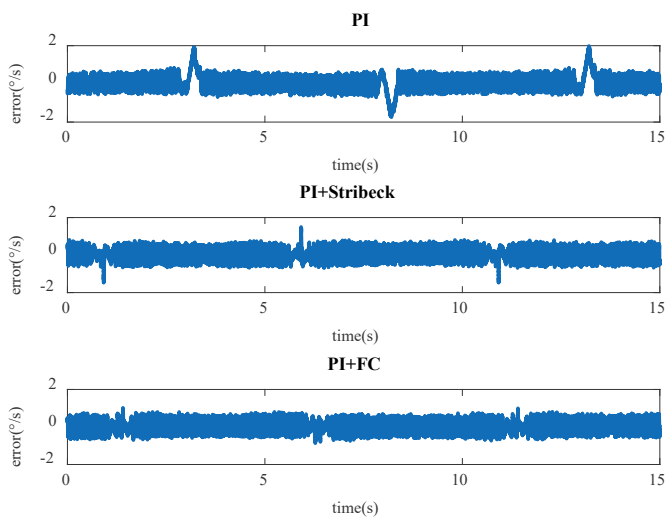

(b)

Figure 13 The actual speed and speed tracking error using $\mathrm{PI}, \mathrm{PI}+\mathrm{Stribeck}$ and $\mathrm{PI}+\mathrm{FC}$ as the speed controller when the amplitude of reference sinusoidal speed is $20 \%$, and the frequency is $0.1 \mathrm{~Hz}$.

Table 3 The zero-crossing speed errors using $\mathrm{PI}, \mathrm{PI}+$ Stribeck and $\mathrm{PI}+\mathrm{FC}$ as the speed controller

\begin{tabular}{|c|c|c|c|}
\hline Control method & PI & PI+Stribeck & PI+FC \\
\hline Zero-crossing speed error $(\%)$ & 2.0 & 1.6 & 1.0 \\
\hline
\end{tabular}

As can be seen from Table 3, in comparison with the control method of PI+Stribeck, the zero-crossing speed error can be reduced by $37.5 \%$ using $\mathrm{PI}+\mathrm{FC}$ as the speed controller, which indicates that the proposed friction model also has a higher accuracy in pre-sliding regime.

According to above results, the proposed friction model behaves better than the classical one both in presliding and sliding regime due to the fact that the proposed model considers both angular distribution and rotating speed while the classical Stribeck model only takes rotating speed into consideration.

\subsection{Discussion}

In order to illustrate the effect of the proposed friction model in improving motion performance furthermore, compare the low-speed-motion smoothness and speed tracking accuracy by three speed control methods, i.e., PI with the proposed friction model-based compensation (PI+FC), PI with disturbance observer compensation (PI+DOB), and PI with both friction model-based feedforward and disturbance observer compensation
$(\mathrm{PI}+\mathrm{FC}+\mathrm{DOB})$, respectively. The control diagrams are shown in Fig. 14.

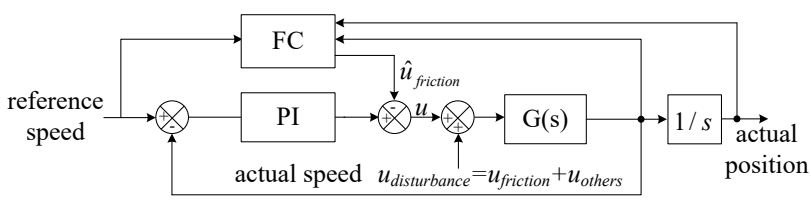

(a)

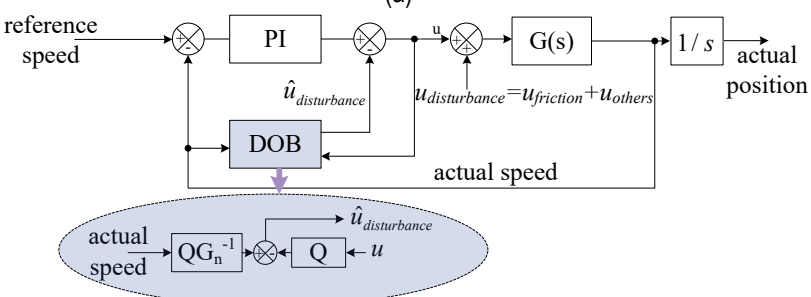

(b)

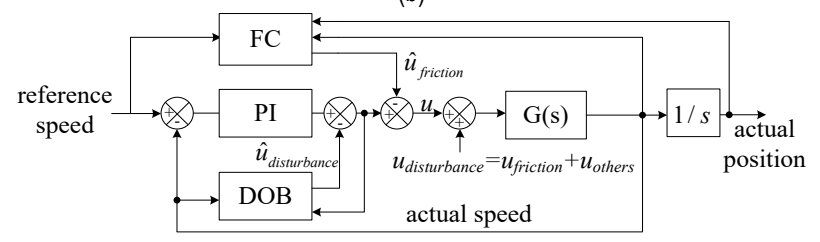

(c)

Figure 14 Diagrams of three control methods: (a) PI+FC; (b) PI+DOB; (c) $\mathrm{PI}+\mathrm{FC}+\mathrm{DOB}$ 


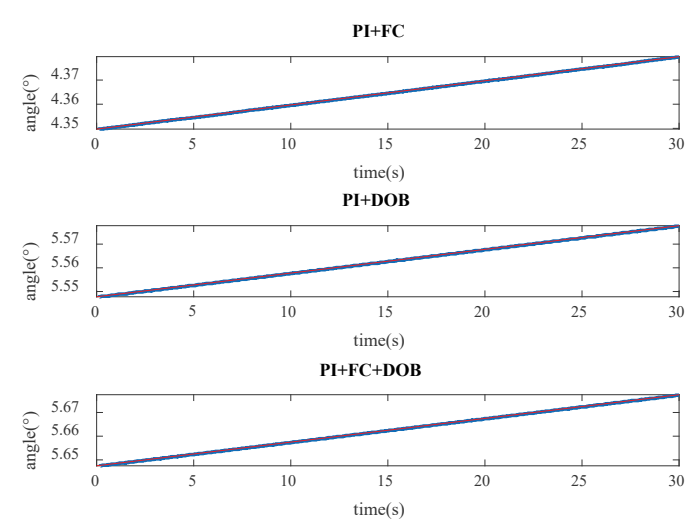

(a)

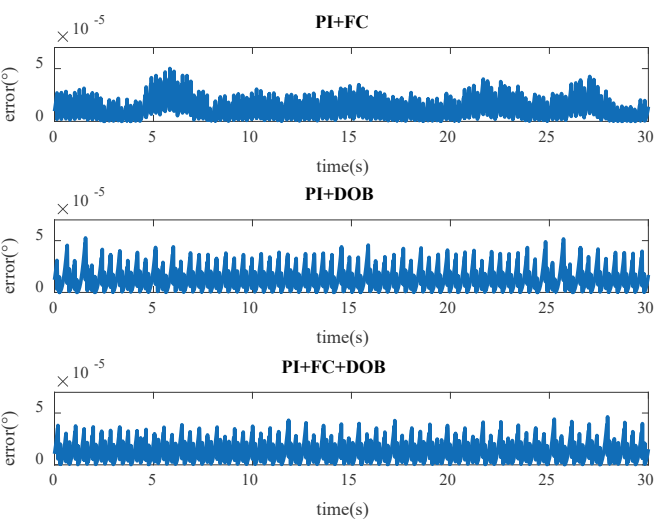

(b)

Figure 15 The actual position and angular tracking error using $\mathrm{PI}+\mathrm{FC}, \mathrm{PI}+\mathrm{DOB}$ and $\mathrm{PI}+\mathrm{FC}+\mathrm{DOB}$ as the speed controller (the slope of the reference position $r=0.001 \%$ s).

Table 4 The angular fluctuation rate using $\mathrm{Pl}+\mathrm{FC}, \mathrm{PI}+\mathrm{DOB}$ and $\mathrm{PI}+\mathrm{FC}+\mathrm{DOB}$ as

\begin{tabular}{|c|c|c|c|}
\hline the speed controller \\
\hline Control method & $\mathrm{PI}+\mathrm{FC}$ & $\mathrm{PI}+\mathrm{DOB}$ & $\mathrm{PI}+\mathrm{FC}+\mathrm{DOB}$ \\
\hline Angular fluctuation rate $\varepsilon(\%)$ & 16.67 & 13.33 & 10.00 \\
\hline
\end{tabular}

Construct an angular close loop in the motor side, and set a ramp signal with the slope $r=0.001 \%$ as the reference position. Then sample the angle value from the incremental encoder and calculate the tracking error, as is

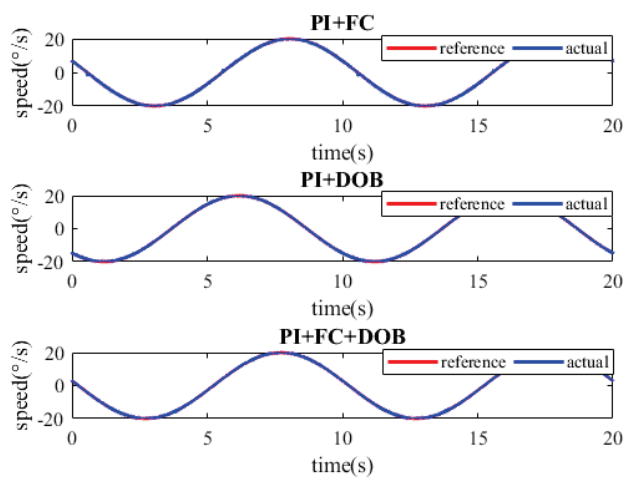

(a) shown in Fig. 15. Thus the angular fluctuation rate $\varepsilon$ can be obtained by substituting the tracking error into (13). The results are listed in Tab. 4.

As can be seen from Fig. 15 and Tab. 4, the best lowspeed-motion smoothness is achieved by $\mathrm{PI}+\mathrm{FC}+\mathrm{DOB}$ with the angular fluctuation rate $\varepsilon$ of only $10.00 \%$. Meanwhile, $\varepsilon$ raises to $13.33 \%$ when taking $\mathrm{PI}+\mathrm{DOB}$ as the speed controller, and reaches up to $16.67 \%$ when using $\mathrm{PI}+\mathrm{FC}$.

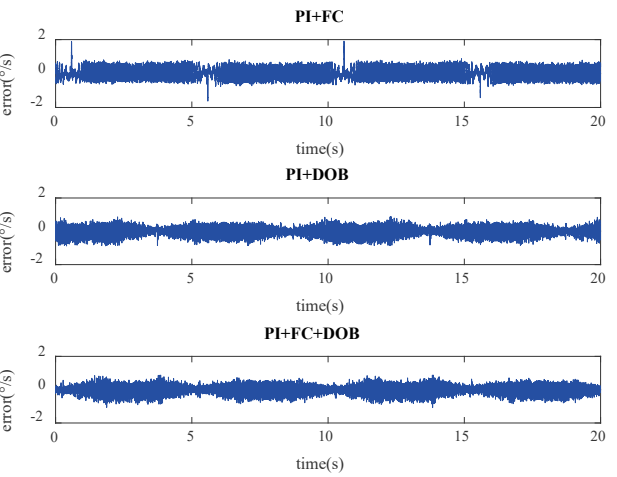

(b)

Figure 16 The actual speed and speed tracking error using $\mathrm{PI}+\mathrm{FC}, \mathrm{PI}+\mathrm{DOB}$ and $\mathrm{PI}+\mathrm{FC}+\mathrm{DOB}$ as the speed controller (the amplitude of reference sinusoidal speed is $20 \%$, and the frequency is $0.1 \mathrm{~Hz}$ ).

Construct a speed close loop in motor side with $\mathrm{PI}+\mathrm{FC}, \mathrm{PI}+\mathrm{DOB}$ and $\mathrm{PI}+\mathrm{FC}+\mathrm{DOB}$ as the speed controller respectively, and make a sinusoidal signal with an amplitude of $20 \%$ and a frequency of $0.1 \mathrm{~Hz}$ as the reference speed. The speed tracking errors by the three control methods can be shown in Fig. 16, and the zerocrossing speed errors are listed respectively in Tab. 5 .

Table 5 The zero-crossing speed errors using $\mathrm{PI}+\mathrm{FC}, \mathrm{PI}+\mathrm{DOB}$ and $\mathrm{PI}+\mathrm{FC}+\mathrm{DOB}$ as the speed controller

\begin{tabular}{|c|c|c|c|}
\hline Control method & PI+FC & PI+DOB & PI+FC+DOB \\
\hline Zero-crossing speed error $(\%)$ & 2 & 0.8 & 0.3 \\
\hline
\end{tabular}

In Tab. 5, the zero-crossing speed error is the largest when taking $\mathrm{PI}+\mathrm{FC}$ as the speed controller. However, the error decreases obviously when using $\mathrm{PI}+\mathrm{DOB}$ and reduces to minimum when using $\mathrm{PI}+\mathrm{DOB}+\mathrm{FC}$.

As can be concluded from both Tab. 4 and Tab. 5, taking $\mathrm{PI}+\mathrm{FC}+\mathrm{DOB}$ as the speed controller can achieve the best low-speed-motion smoothness and the minimum zerocrossing speed error. The reason is that PI, as a linear control scheme, mainly regulates the linear dynamics of the
RVTS. Though FC can compensate for the nonlinear friction accurately, it cannot deal with any other nonlinear dynamics, such as backlash and hysteresis, in the assembly. As another type of control method, DOB performs observation and compensation for disturbances with usage of the nominal model $G_{\mathrm{n}}$. Since DOB does not distinguish between the disturbance types, it has a wider range of suppression capabilities than FC. Therefore, PI+DOB performs better than $\mathrm{PI}+\mathrm{FC}$ in assembly with multiple nonlinear dynamics. However, the performance of DOB depends on the accuracy of the nominal model. Moreover, the difference between nominal model and actual assemble cannot be reduced unless the main nonlinear dynamics of the RVTS, such as friction, is compensated. As a result, $\mathrm{PI}+\mathrm{FC}+\mathrm{DOB}$ achieves a better motion performance than $\mathrm{PI}+\mathrm{DOB}$.

\section{CONCLUSIONS}

A novel angular position-based two-stage friction model for RV transmission system has been proposed and 
validated in this paper. The pre-sliding friction model with a relatively high accuracy has been achieved in accordance with the maximum elastic deformation recovery angle, and the novel sliding friction model based on the angularsegmented Stribeck model has shown a satisfactory accuracy as well. The classical Stribeck model and the proposed model have been used for friction compensation respectively, and the motion performances have been compared. The results show that when using the proposed model, the low-speed-motion smoothness of the RVTS can be improved by $14.2 \%$, and the speed tracking accuracy can be improved by $37.5 \%$, which indicates that the novel modeling and compensation methods proposed in this paper can effectively improve the servo performance of RVTS. However, since the proposed model can only be used to compensate friction, it seems difficult to achieve a better motion performance for the actual RVTS working with multiple nonlinear factors, such as friction, backlash and structural flexibility. So in order to break through the above limitation, a further research on the accurate dynamic modeling for RVTS will be undertaken later.

\section{REFERENCES}

[1] Armstrong, H. B., Dupont, P., \& Wit, C. C. D. (1994). A survey of models, analysis tools and compensation methods for the control of machines with friction. Automatica, 30(7), 1083-1138. https://doi.org/10.1016/0005-1098(94)90209-7

[2] Freidovich, L., et al. (2006). Friction compensation based on LuGre model. In Proceedings of the $45^{\text {th }}$ IEEE Conference on Decision and Control, San Diego, CA, USA, 3837-3842. https://doi.org/10.1109/CDC.2006.376780

[3] Lampaert, V., Albender, F., \& Swevers, J. (2003). A generalized Maxwell-slip friction model appropriate for control purposes. In 2003 IEEE International Workshop on Workload Characterization, Saint Petersburg, Russia, 11701177. https://doi.org/10.1109/PHYCON.2003.1237071

[4] Lv, M. S. (2016). Simulation and experimental study of transmission characteristic of RV reducer. MSc thesis, School of Mechatronics Engineering, Harbin Institute of Technology, Harbin, China.

[5] Wu, W. X. (2013). Joint friction analysis and low-speed high-precision motion control of multi-DOF serial robots. PhD dissertation, School of Mechanical Engineering, Zhejiang Univ, Hangzhou, China.

[6] Yu, D., et al. (2017). Theoretical calculation and analysis on friction torque in RV reducer main bearing. Journal of Zhejiang University (Engineering Science), 51(10), 19281936. https://doi.org/10.3785/j.issn.1008-973X.2017.10.006

[7] Xue, H. (2017). Tribological analysis of needle roller bearings in RV reducer. MSc thesis, School of Mechanical Engineering, Zhejiang Univ, Hangzhou, China.

[8] Jiang, K., Zhu, C., \& Tang, M. (2012). A Uniform Control Method for Imbalance Compensation and Automation Balancing in Active Magnetic Bearing-Rotor Systems. $J$. Dyn. Sys., Meas., Control., 134(2), 021006. https://doi.org/10.1115/1.4005279

[9] Li, X. P., et al. (2012). Review and Prospect of the Research of Vibration and Friction Mechanism of Machine Joint Surfaces. Applied Mechanics and Materials, 121-126, 34873492. https://doi.org/10.4028/www.scientific.net/AMM.121-126.3487

[10] Yen, J., Huang, S., \& Lu, S. (1999). A New Compensator for Servo Systems with Position Dependent Friction. J. Dyn. Sys., Meas., Control., 121(4), 612-618. https://doi.org/10.1115/1.2802524
[11] Wordena, K., Wonga, C., Parlitzb, U., et al. (2007) Identification of pre-sliding and sliding friction dynamics: Grey box and black-box models. Mechanical Systems and Signal Processing, 21(1), 514-534. https://doi.org/10.1016/j.ymssp.2005.09.004

[12] Taghirad, H. D. \& Belanger, P. R. (1998). Modeling and parameter identification of harmonic drive systems. J Dyn Syst-T ASME, 120(4), 439-444. https://doi.org/10.1115/1.2801484

[13] Cong, S., et al. (2016). Isolation control for inertially stabilized platform based on nonlinear friction compensation. Nonlinear Dynamics, 84(3), 1123-1133. https://doi.org/10.1007/s11071-015-2557-4

[14] Li, F. T., et al. (2017). Friction identification and compensation design for precision positioning. Adv. Manuf., 5(2), 120-129. https://doi.org/10.1007/s40436-017-0171-z

[15] Liao, H. B., Fan, S. X., \& Fan, D. P. (2016). Friction compensation of harmonic gear based on location relationship. P I Mech Eng I-J Sys, 230(8), 695-705. https://doi.org/10.1177/0959651816650083

[16] Yang, M., et al. (2017). A two-stage friction model and its application in tracking error pre-compensation of $\mathrm{CNC}$ machine tools. Precis Eng, 51. https://doi.org/10.1016/j.precisioneng.2017.09.014

[17] Aung, M. T. S., Kikuuwe, R., \& Yamamoto, M. (2014). Friction compensation of geared actuators with high presliding stiffness. J. Dyn. Sys., Meas., Control, 137(1), 011007. https://doi.org/10.1115/1.4027503

[18] Tan, W., et al. (2012). Friction compensation based on LuGre model with modified viscous friction. Journal of Tianjin University, 45(9), 824-828. https://doi.org/10.1109/CDC.2006.376780

[19] Jin, R., Chen, W., \& Simpson, T. (2001). Comparative studies of metamodelling techniques under multiple modelling criteria.Struct Multidisc Optim, 23(01), 1-13. https://doi.org/10.1007/s00158-001-0160-4

\section{Contact information:}

Chao Qi

Corresponding author

College of Artificial Intelligence,

National University of Defense Technology, Deya Road No. 109, Kaifu District,

Changsha 410073, China

Email: nudtqichao@yeah.net

\section{Xianliang Jiang}

College of Artificial Intelligence,

National University of Defense Technology, Deya Road No. 109, Kaifu District, Changsha 410073, China

\section{Hongbo Liao}

Northwest Institute of Nuclear Technology,

Pingyu Road No. 28, Lingtong District, Xi'an 710024, China

\section{Dapeng Fan}

College of Artificial Intelligence

National University of Defense Technology,

Deya Road No. 109, Kaifu District, Changsha 410073, China

\section{Sheng Zhang}

College of Information and Communication,

National University of Defense Technology,

Jiefanggongyuan Road No. 45, Jiang'an District, Wuhan 430010, China 